\title{
Status and future developments of integrated photonic spectrographs for astronomy and Earth and planetary sciences
}

Jovanovic, N., Cvetojevic, N., Daal, M., Mazin, B., Moreira, R., et al.

N. Jovanovic, N. Cvetojevic, M. Daal, B. Mazin, R. Moreira, D. Mawet, G. Vasisht, C. Beichman, K. Wallace, J. Jewell, S. Leifer, R. Dekany, M. Porter, "Status and future developments of integrated photonic spectrographs for astronomy and Earth and planetary sciences," Proc. SPIE 11287, Photonic Instrumentation Engineering VII, 112870K (2 March 2020); doi: $10.1117 / 12.2546959$

SPIE. Event: SPIE OPTO, 2020, San Francisco, California, United States 


\title{
Status and future developments of integrated photonic spectrographs for astronomy and Earth \& planetary sciences
}

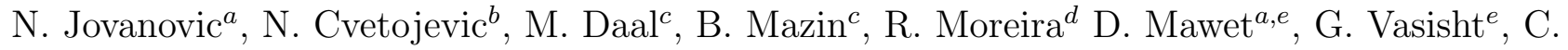 \\ Beichman $^{a, e}$, K. Wallace ${ }^{e}$, J. Jewell ${ }^{e}$, S. Leifer ${ }^{e}$, R. Dekany ${ }^{a}$, M. Porter ${ }^{a}$ \\ ${ }^{a}$ Department of Astronomy, California Institute of Technology, 1200 E. California Blvd., \\ Pasadena, CA, 91125, USA; \\ ${ }^{b}$ Laboratoire Lagrange, Observatoire de la Côte d'Azur, Université Côte d'Azur, 06304 Nice, \\ France \\ ${ }^{c}$ Department of Physics, University of California, Santa Barbara, California 93106, USA \\ ${ }^{d}$ Ultra-Low Loss Technologies, USA \\ ${ }^{e}$ Jet Propulsion Laboratory, California Institute of Technology, 4800 Oak Grove Drive, \\ Pasadena, CA, 91109, USA;
}

\begin{abstract}
The size and cost of astronomical instruments for extremely large telescopes (ELTs), are pushing the limits of what is feasible, requiring optical components at the very edge of achievable size and performance. Operating at the diffraction-limit, the realm of photonic technologies, allows for highly compact instruments to be realized. In particular, Integrated Photonic Spectrographs (IPSs) have the potential to replace an instrument the size of a car with one that can be held in the palm of a hand. This miniaturization in turn offers dramatic improvements in mechanical and thermal stability. Owing to the single-mode fiber feed, the performance of the spectrograph is decoupled from the telescope and the instruments point spread function can be calibrated with a much higher precision. These effects combined mean that an IPS can provide superior performance with respect to a classical bulk optic spectrograph.

In this paper we provide a summary of efforts made to qualify IPSs for astronomical applications to date. These include the early characterization of arrayed waveguide gratings for multi-object injection and modifications to facilitate a continuous spectrum, to the integration of these devices into prototypical instruments and most recently the demonstration of a highly optimized instrument directly fed from an 8-m telescope. We will then outline development paths necessary for astronomy, currently underway, which include broadening operating bands, bandwidth, increasing resolution, implementing cross-disperison on-chip and integrating these devices with other photonic technologies and detectors such as superconducting Microwave Kinetic Inductance Detector arrays. Although the focus of this work is on IPS applicability to astronomy, they may be even more ideally suited to Earth \& planetary science applications.
\end{abstract}

Keywords: Integrated photonic spectrographs, arrayed waveguide gratings, astronomy, astronomical instruments, miniturization

\section{INTRODUCTION}

Spectroscopy is an extremely powerful analytical tool that has been widely used in astronomy. It allows for the determination of the chemical composition of an astrophysical target, the abundances of its constituents, the velocity of the object relative to the Earth and the distance to the target. Offering such rich insights, it has been applied to a broad range of fields in astronomy including the detection of exoplanets via the Doppler effect, ${ }^{1}$ studying the black hole at the center of the Milky way and creating a 3D map of the galaxies in the Universe, to name a few. ${ }^{2}$

Further author information: (Send correspondence to Nemanja Jovanovic)

Nemanja Jovanovic: E-mail: jovanovic.nem@gmail.com, Telephone: +1 (626) 3951214

Photonic Instrumentation Engineering VII, edited by Yakov Soskind, Lynda E. Busse, Proc. of SPIE

Vol. 11287, 112870K · C 2020 SPIE · CCC code: 0277-786X/20/\$21 · doi: 10.1117/12.2546959 
Each science case imposes a unique set of requirements on the spectrograph. For example, precision radial velocity detections of exoplanets requires a high resolving power $(>50,000)$ to be able to resolve the spectral features, increasing spectral content and maximizing the precision of detection. On the other hand, spectral typing of stars, can typically be done at much lower resolving power $(<5,000)$. Given the aim is to always maximize the use of the relatively expensive detector pixels for a given science case, there is no single instrument design that meets all requirements for a range of applications and hence astronomical spectrographs come in a variety of shapes and sizes and are typically customized for each case. This is compounded by the fact that the instrument specifications depend on the telescope and site properties. To understand this, consider the basic equations governing spectrograph performance. Equations 1 and 2 show the resolution $(\delta \lambda)$ and resolving power $(R)$ of a spectrograph, respectively. ${ }^{3}$

$$
\begin{gathered}
\delta \lambda=\frac{r \phi D_{\mathrm{Tel} .}}{A d_{\mathrm{col} .}} \\
R=\frac{\lambda}{\delta \lambda}=\frac{\lambda A d_{\mathrm{col} .}}{r \phi D_{\mathrm{Tel}} .}
\end{gathered}
$$

The parameters in the equations are defined as follows: $\lambda$ is the wavelength of light, $r$ is the anamorphic factor, $\phi$ is the angular slit size, $D_{\text {Tel. }}$ is the diameter of the telescope, $A$ is the angular dispersion, and $d_{\text {col. }}$ is the diameter of the collimated beam incident on the disperser. It can be seen that the resolving power is inversely dependent on the slit size and is maximized when the slit size is at a minimum (i.e. the diffraction-limit). For a lot of existing telescopes, which operate in the seeing-limit (where the smallest spot size that can be formed is limited by the atmospheric turbulence at the site and not the telescope diameter) and hence require a larger slit size, a given resolving power can only be maintained by increasing the size of the collimator optic (for a fixed wavelength and grating choice). In addition, the resolving power will decrease as the telescope diameter increases. For a given wavelength, angular dispersion, slit size, and anamorphic factor, the diameter of the collimator (and hence disperser and camera optics) needs to proportionally increase to maintain a certain resolving power. To preserve the focal ratio of the collimator and the magnification of the spectrograph, the focal length of both the collimator and camera optics must also increase proportionally. This ultimately drives an increase in the volume of the entire seeing-limited spectrograph and explains why such a spectrograph must be tailored for the science case, telescope and site. ${ }^{4}$.

However, by operating at the diffraction-limit, the slit size can be expressed as

$$
\phi \approx \frac{\lambda}{D_{\text {Tel. }}} .
$$

Substituting Equation 3 into Equations 1 and 2, we obtain

$$
\delta \lambda=\frac{r \lambda}{A d_{\mathrm{col}}}
$$

and

$$
R=\frac{\lambda}{\delta \lambda}=\frac{A d_{\text {col. }}}{r}
$$

In the diffraction-limited regime, it is clear that both $\delta \lambda$ and $R$ are now independent of the telescope diameter and are only a function of the optics in the spectrograph itself (i.e., the angular dispersion and collimated beam size). This means that a high-resolution spectrograph, which exploits a diffraction-limited feed would have the same properties independent of the site, and the instrument can be designed without consideration of the telescope it will be deployed at. It also means that the volume of the spectrograph no longer scales with telescope aperture for a given resolving power, and very high resolution spectrographs can be made extremely compact. Furthermore, thermal and vibration deflections, which scale with the cube of the length of the instrument, are also significantly reduced, providing exceptional instrument stability. These advantages were first exploited by the RHEA, ${ }^{5}$ iLocator $^{6}$ and PARVI ${ }^{7}$ instruments and more recently the upcoming HISPEC/MODHIS spectrographs. ${ }^{8}$ HISPEC/MODHIS is a SMF-fed spectrograph operating from $\mathrm{y}-\mathrm{K}$ band designed for operation at $R>100,000$ at both the Keck (10-m) and TMT (30-m) Observatories. By operating at the diffraction limit this instrument can operate at both observatories with the same specification 
and will cost several times less than other first-light seeing-limited instruments under development for ELT's. In addition, the design of these instruments can be re-used by other observatories for similar science cases in the future, greatly reducing development costs.

To operate at the diffraction-limit the telescope most either be small compared to the local Fried parameter, be equipped with an adaptive optics ( $\mathrm{AO}$ ) system that corrects for the perturbations injected by the atmosphere, operate at longer wavelengths or be located in space free from the effects of the Earth's atmosphere. For most of the instruments listed above, it is a combination of an advanced AO system combined with operation at longer wavelengths in the near-IR $(1-5 \mu \mathrm{m})$, which enables the spectrograph to operate at the diffraction-limit. Once a diffraction-limited point-spread function (PSF) can be realized, the light can also be injected into a single-mode fiber (SMF) and routed to the psuedo-slit of the spectrograph, allowing it be located remote to the telescope.

Owing to the single spatial mode supported by a SM waveguide, the output of such a device does not evolve with time or with input beam illumination fluctuations, and so is extremely stable. This effect is termed "spatial filtering" and is analogous to passing a beam through a very small pinhole. SMF use offers a clear advantage for instruments that require a stable instrument PSF. For example, it would eliminate modal noise as well as pointing-induced illumination fluctuations common to multimode fiber-fed radial velocity spectrographs. A stable instrument response function makes it possible to calibrate data to a greater precision improving SNR for a given observation. It also means that the performance of the instrument is entirely decoupled from the performance of the telescope, AO system, and injection, which can greatly relax constraints on the design of the telescope and upstream optics. This decoupling reduces both cost and complexity, powerful motivators that have led future space missions such as EarthFinder ${ }^{9}$ to plan for use of SM fibers to detect and study Earth-analogs in the habitable zone around G-stars from space.

By utilizing a SMF to route the light to the spectrograph, interfacing with technologies such as integrated photonic spectrographs is trivial and exploiting such technologies for diffraction-limited spectroscopy becomes an obvious choice.

\section{THE PHOTONIC SPECTROGRAPH}

An Integrated Photonic Spectrograph (IPS) is a compact device, typically measuring a few square centimeters, which consists of an optical circuit imprinted into a transmissive material on a wafer. An example of a device with an $R$ of $\sim 7000$ operating around $1.55 \mu \mathrm{m}$ is shown in Figure 1. Owing to the fact they are fed with a diffraction-limited SMF, these devices include the equivalent of a spectrograph collimator, disperser, and camera optics in a single monolithic element, which fits in the palm of your hand, enabling an order of magnitude reduction in each of the 3 linear instrument dimensions ( 3 orders of magnitude smaller volume) with respect to a bulk-optic counterpart.

Several IPS variants exist including Planar Concave Gratings and Arrayed Waveguide Gratings (AWGs) to name a few (please see ${ }^{12}$ for a review on the topic). Given that AWGs are the most mature versions of IPSs, development and testing for astronomical applications has almost exclusively been reserved to them and hence they form the focus of the discussion in this paper. They were originally developed by the telecommunications industry and are now used in optical networks worldwide. Off-the-shelf devices like the one shown in Figure 1 have been optimized to operate around $1550 \mathrm{~nm}$, with high throughput (78\%), a free-spectral range (FSR) of $50 \mathrm{~nm}$ (defined as the wavelength span of a single order of the dispersion element), and medium $R \sim 7000 .{ }^{13}$ More recently there have been efforts to extend the operating range of such devices for biomedical applications. ${ }^{14}$ This milestone demonstration successfully realized an AWG that could operate from 400-1700 nm in a single shot at low resolving power $(R \sim 100)$.

\subsection{Astronomical Developments of Integrated Photonic Spectrographs to Date}

Bland-Hawthorn et al. were one of the first to realize the potential of IPSs for astronomy ${ }^{15}$ and later outlined the PIMMS concept, ${ }^{16}$ a vision for an all photonic instrument that combined photonic lanterns, fiber Bragg gratings and AWGs. It wasn't until 2009 that astronomers began playing with commercial-off-the-shelf (COTS) devices. ${ }^{10}$ The COTS device mentioned above, shown in Fig. 1 was first used to generate a full H-band spectrum of night time airglow by pointing the collection SMF towards the sky (no optics or telescope) and using a bulk 


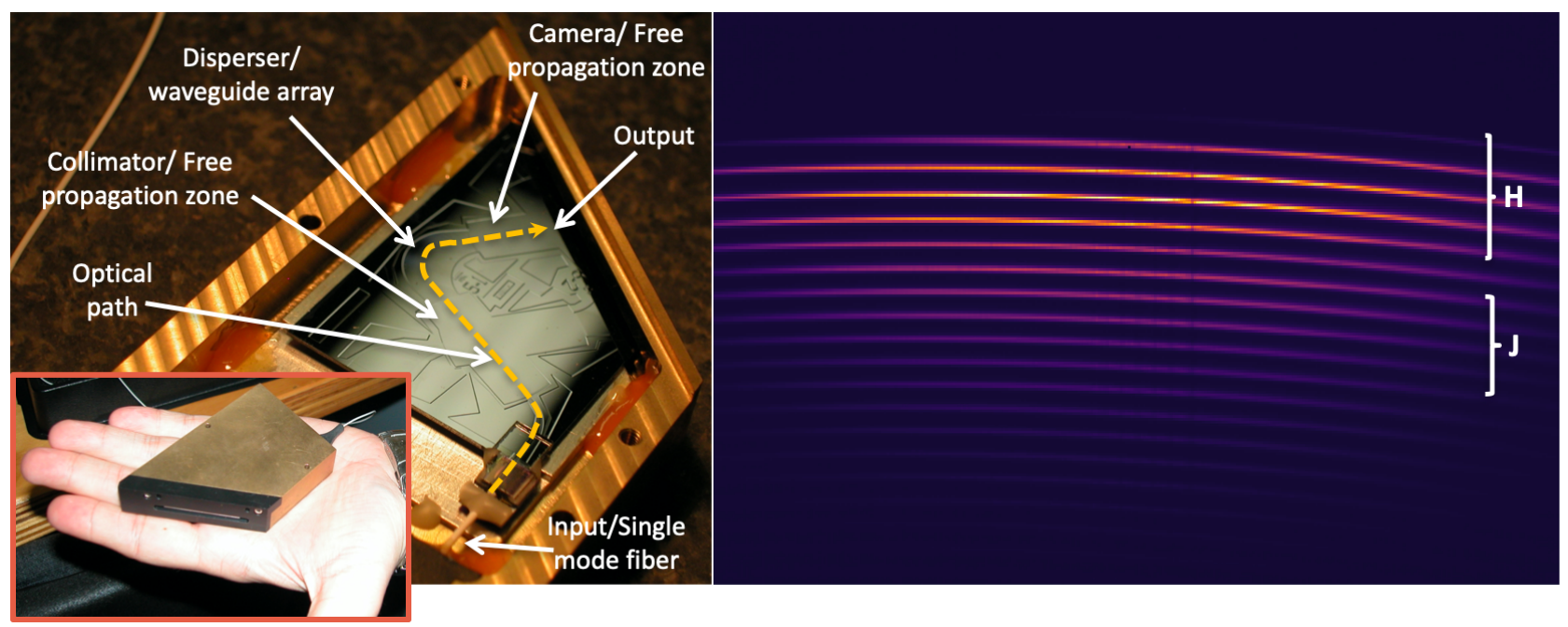

Figure 1. (Left) An arrayed waveguide grating version of an IPS device. An off-the-shelf device with a $R=7000$, packaged and connected to a SMF feed. The circuit on the chip is clearly visible. ${ }^{10}$ (Inset) An image for sense of scale. Disclaimer, to the best of the teams knowledge the hand used in this image is of average size. (Right) A J and $\mathrm{H}$ band spectrum of a lamp taken with the AWG prototype instrument in the laboratory. ${ }^{11}$

optic cross-disperser to unravel the orders. ${ }^{10}$ The team next showed that the resolving power of the AWG could be improved by removing the input tapers and that a continuous spectrum could be obtained by removing the output waveguides. In addition, they demonstrated that it was possible to use an AWG to process signals from multiple SMFs simultaneously in a single chip. The key was to use bulk optic cross-dispersion at the output of the chip. ${ }^{13}$ This is highly desirable as either the light from multiple objects, the outputs of a photonic lantern or a star and calibration source can be acquired at the same time. This concept was tested at the Australian Astronomical Telescope (AAT). ${ }^{17}$ For this test the GNOSIS ${ }^{18}$ injection was used. The Injection consisted of 7 micro-lenses, which sample the seeing-limited PSF of the AAT, and couple the light to $7,19 \times 1$ photonic lanterns. ${ }^{19,20}$ A photonic lantern has a multimode input waveguide allowing for efficient coupling from the telescope PSF, which is reformatted to many, in this case 19 SMFs. The AWG could only accept 12 SMFs, so 12 out of 19 SMFs from one of the 7 photonic lanterns were used, making the injection extremely inefficient, but sufficient to test spectral extraction from multiple input fibers on-sky. With this setup, the team collected the first stellar spectra with an IPS and managed to clearly observe the CO lines in the spectrum of the star $\pi 1$ Gru.

To take full advantage of the technology, a dedicated SMF injection unit was built behind the extreme AO system, SCExAO at the 8-m Subaru Telescope. With > 50\% coupling efficiency from an 8-m telescope on the ground directly into a SMF realized, ${ }^{21}$ it was possible to take the ultimate step and directly feed the IPS device. The team used the spectrograph once again with external cross-dispersion to enable operation across the $\mathrm{J}$ and $\mathrm{H}$-bands with a total throughput from fiber to detector of $42 \pm 3 \%$ and a resolving power of $\sim 5000(5 \%$ throughput from sky-to-detector assuming a $65 \%$ Strehl ratio). ${ }^{11}$ The photonic instrument successfully recovered spectra from several stellar sources with similar quality and SNR as those collected by existing spectrographs. This demonstration shows that it is now possible to consider a fully photonic instrument that can be competitive with traditional approaches on large telescopes.

Although this initial success was encouraging, the demonstration device lacked the specifications suited to most astronomical applications. Having proven the fundamental advantages, it was clear the next step was to develop custom IPS designs for astrophysics applications.

In 2010, Lawrence et al. explored the equations that govern the performance of an AWG device outlining how to modify one to obtain a specific set of properties. ${ }^{22}$ This word has been used by several groups to try to customize devices for specific applications.

A group at the University of Maryland have begun developing AWGs in the SiN platform, in-house. SiN is 
a higher index contrast material that allows much tighter bends and hence the overall footprint of the AWG to be greatly reduced compared to Silica-on-Silicon (the platform for the COTS device mentioned above). The advantage of developing the devices in-house is that the team retains the knowledge of the design and fabrication process, greatly reducing costs on future devices and/or instruments that utilize these chips. However, this also requires the team to master the fabrication process, which is challenging. Initial results include a demonstration of a device with $23 \%$ throughput, which operates from 1450-1650 nm, with an $R \sim 1,300$ on a single linear polarization. ${ }^{23}$ While work is ongoing to improve the performance of these devices, the group have also tried to address the difficulty of coupling into the the high-index-contrast waveguides. To this end they have demonstrated $>90 \%$ coupling from an ultra-high numerical aperture fiber to the input waveguide ${ }^{24}$ and a highly efficient taper to convert from the weakly guided input waveguide to a strongly guided AWG feed waveguide. In addition, the team has developed waveguide Bragg gratings, which can be integrated onto the chip with the IPSs to spectrally filter for $\mathrm{OH}$ suppression applications, ${ }^{25}$ for example.

An alternative approach to developing integrated photonics is to use ultrafast laser inscription (ULI). ULI utilizes focused femtosecond laser pulses to modify the refractive index of a transparent dielectric, such as a glass or crystal material. The benefit of the technique is that its $3 \mathrm{D}$ compliant and relatively easy and fast to prototype devices as a mask is not required. A group at Macquarie University has applied this technique to realize AWGs operating in the visible. The team initially focused on producing slab waveguides for the free propagation zones and achieved a standard deviation in the refractive index of $2 \%$, as well as demonstrating efficient adiabatic tapers to efficiently couple to them. ${ }^{26}$ They then developed a full AWG device that operated around $632.8 \mathrm{~nm}$, with a free spectral range of $22 \mathrm{~nm}$ and a $R \sim 470 .{ }^{27}$ As a second step, the AWG was fed with a 3 ports from an integrated photonic lantern inscribed on the same chip and showed it performed as expected from simulation. The concept was extended to combine the integrated photonic lantern with 3 AWGs stacked vertically on a single chip. ${ }^{28}$ The output of the 3 AWGs was interfered on the final detector to realize a spectro-interferometer, which can be used for advanced imaging applications. Although the devices are not at the point where they could be exploited to realize efficient astronomical instruments, this opens up a new avenue to rapidly prototyping astrophotonic spectrographs with the ability to easily integrate other technologies on the same chip.

\subsection{Next steps in IPS Technology Development}

Given AWG technology is well understood, has been tested and characterized over the past 10 years and has demonstrated favorable properties for application in astronomy, it is the ideal choice for the next stage of optimization (although other technologies should be explored in parallel). AWGs should be developed beyond the requirements for the telecommunications industry to support the wide ranging scope of astronomical applications, by engineering them for higher spectral resolution $(R>7000)$ than is currently available, in entirely new wavebands spanning from $500 \mathrm{~nm}$ in the visible to $5.0 \mu \mathrm{m}$ in the MIR for use in both single and multi-object science cases with on-chip cross-dispersion implemented.

Figure 2 shows a custom designed AWG in the silica-on-silicon platform, which aims to address the push to higher resolution. ${ }^{29}$ This design could deliver a device capable of a $R \sim 65,000$, while operating from $0.95 \mu \mathrm{m}$ to $1.78 \mu \mathrm{m}$ (from the astronomical $\mathrm{y}-\mathrm{H}$ bands) with an average theoretical throughput of $60 \%$. Each data point in the figure represents a different FSR, indicating that the bandwidth of each FSR ranges from 5-20 nm. To separate the orders at the output and extend the spectral bandwidth of the device, bulk optic cross-dispersion would need to be implemented. However, it is possible to achieve cross-dispersion on a chip. The most commonly used method is using either cascaded or tandem AWGs. In this scenario, a low resolution, large bandwidth AWG is used to first split the spectrum into a number of discrete channels, which are then fed into a series of higher resolution AWGs. The output waveguides of these devices are then all routed to the same side of the chip to be extracted for analysis. This approach eliminates the bulk optic cross-dispersor, but may make it difficult to realize a continuous spectrum, although a discrete spectrum may also be useful. The design shown in Fig. 2 could be reworked to include cascaded AWGs, which would reduce the complexity of the single step circuit design, which requires almost 1000 carefully positioned waveguides in the array, and eliminate the cross-dispersion optics. Therefore, on-chip cross dispersion can increase the bandwidth of the device greatly, while miniaturizing the foot print of the spectrograph. 

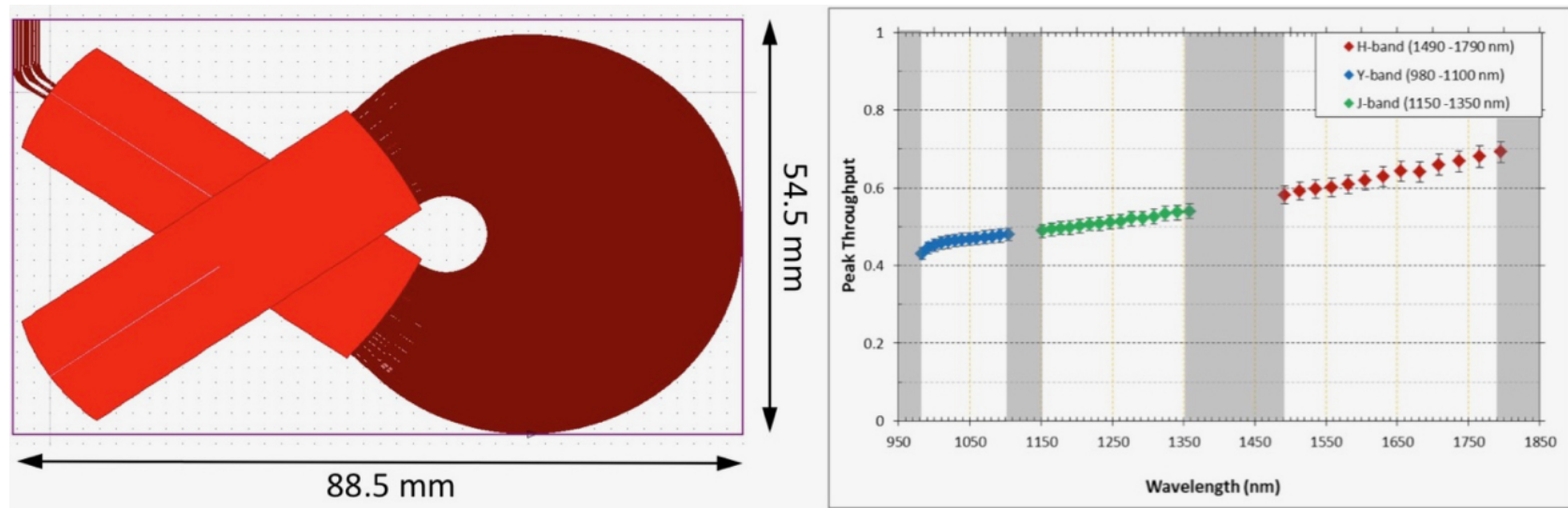

Figure 2. (Left) A custom designed AWG in the silica-on-silicon platform, offering an $R \sim 65,000$ around $1.55 \mu \mathrm{m}$. (Right) Theoretical throughput of the device. ${ }^{29}$

The design of the circuit should be done in consideration of how one plans to extract and image the output signals on a detector. The most streamlined concept involves butting the output facet of an AWG directly against the detector, eliminating the need for reimaging optics (see top panel in Fig. 3). Since the output is formatted in a 1D line, a linear array detector could be used reducing costs and complexity. This approach is currently being pursued by a team at UCSB in collaboration with the company Ultra-Low Loss Technologies *. In this particular project, a superconducting detector known as a Microwave Kinetic Inductance Detector(MKID) is being used, which not only offers noiseless detection of photons, but can also distinguish their energy, albeit at low resolving power $\left(R \sim 20\right.$ in the visible band) ${ }^{30}$ In this case, the detector can be used as the cross-dispersor/order sorter reducing the complexity of the AWG design. ${ }^{31}$ The project has only recently commenced, but aims to develop an AWG which operates from 400-800 nm, has a FSR of $\sim 100 \mathrm{~nm}$, with 1024 discrete output channels (output waveguides). The waveguides would be butted against the 1024 pixels of a linear MKID array. Each waveguide would effectively see 4 spectral channels separated by $\sim 100 \mathrm{~nm}$, requiring a cross-dispersion of $>8$ to be able to able to discriminate the photons at the detector, which is easily achieved with current technology ${ }^{32}$ The overall resolving power of the instrument will thus be $\sim 5,000$. The team will use SiN technology and the throughput of the entire instrument, from fiber-to-detector is predicted to be $\sim 60 \%$. This important development will also represent the first time AWGs are tested at very low temperatures (indeed ultra-low temperatures in this case, $100 \mathrm{mK}$ ), and will inform on how compliant these technologies are in those regimes. This unique merger of two technologies really exemplifies the potential of IPS technologies.

There are several methods by which the number of objects that can be observed can be increased. The first approach involves stacking the AWGs on top of one another and utilizing a 2D detector array instead (Figure 3(c)). This assumes that only a single FSR from each device needs to be imaged by the detector. An alternative method to increase the number of objects that can be studied is to feed the AWGs with multiple input SMFs (see Figure 3(b)). To unravel the signals from different sources or increase the bandwidth for a single object beyond a single FSR, the orders need to be separated with a cross-disperser which can be done on-chip as outlined above.

\section{OUTLOOK}

IPS technologies are extremely promising for other fields, especially Earth \& planetary sciences. A large thrust of this field is the detailed spectroscopic study of the atmospheres of the planets in the solar system, carried out from remote satellites. This field is rapidly growing with the demand for Earth monitoring satellites to track climate change. Molecules like methane and carbon dioxide, two of the key species driving climate change can be easily resolved with a spectrograph that can achieve a $R>10,000$ in the NIR $(1.2-1.8 \mu \mathrm{m})$. These requirements only pose a modest challange to the design of a new custom IPSs for these applications. A SMF could be positioned to stare at the scene with no collection optics providing a spot on the target constrained by

\footnotetext{
*https://www.ultralowlosstechnologies.com/
} 


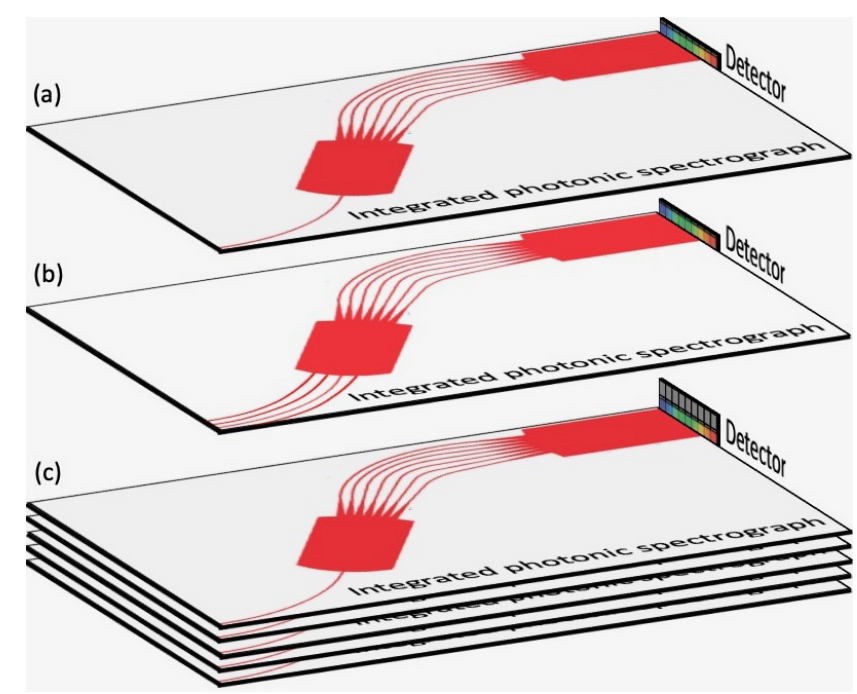

Figure 3. Concept of an AWG instrument (a) AWG butted against a linear detector, (b) AWG with multiple input fibers butted against a linear detector and (d) multiple AWGs butted against a 2 D detector. ${ }^{33}$

the numerical aperture of the fiber. A huge benefit of utilizing AWGs for this application is that once designed, they can mass produced at very low cost. This would enable a cost-effective fleet of CubeSats to be deployed allowing for continuous and global coverage, greatly improving data quantity.

Another area that can amplify the impact of IPSs, is compliance with other photonic technologies. For example, laser frequency combs, fiber Bragg gratings, photonic lanterns, fiber based etalons, are just some of the technologies that can be efficiently interfaced with IPSs, and but improve the overall performance provided by the spectrograph. Owing to their compact nature, they can maintain the already low footprint of the IPS, but offer advanced functionalities. Demonstrating AWGs integrated into larger photonic instruments will no doubt be a focus of development over the coming decade.

\section{SUMMARY}

IPSs promise large gains in the miniaturization of spectrographs, which if applied to astronomy or Earth \& planetary sciences, could have a significant impact. As they operate at the diffraction-limit, they can readily be interfaced with other photonic technologies, offering advanced functionalities. Over the past ten years, progress has been made to understand the limitations of AWG technology and try to optimize it for use in astronomy. The next step is to customize these devices to address specific science cases by driving the devices to higher resolving power, broader bandwidths operation, shifting to new wavebands to name a few. The limits of these technologies is unclear, but their perceived impact is striking.

\section{ACKNOWLEDGMENTS}

N. Cvetojevic acknowledges funding from the European Re-search Council (ERC) under the European Union's Horizon 2020 research andinnovation program (grant agreement CoG - 683029).

\section{REFERENCES}

[1] Mayor, M. and Queloz, D., "A Jupiter-mass companion to a solar-type star," Nature 378, 355-359 (Nov 1995).

[2] Colless, M., Dalton, G., Maddox, S., Sutherland, W., Norberg, P., Cole, S., Bland-Hawthorn, J., Bridges, T., Cannon, R., Collins, C., Couch, W., Cross, N., Deeley, K., de Propris, R., Driver, S. P., Efstathiou, G., Ellis, R. S., Frenk, C. S., Glazebrook, K., Jackson, C., Lahav, O., Lewis, I., Lumsden, S., Madgwick, D., Peacock, J. A., Peterson, B. A., Price, I., Seaborne, M., and Taylor, K., "The 2dF Galaxy Redshift Survey: spectra and redshifts," Monthly Notices of the Royal Astronomical Society 328, 1039-1063 (12 2001). 
[3] Schroeder, D., [Astronomical Optics], Elsevier Science (1999).

[4] Jovanovic, N., Schwab, C., Cvetojevic, N., Guyon, O., and Martinache, F., "Enhancing Stellar Spectroscopy with Extreme Adaptive Optics and Photonics," PASP 128, 121001 (Dec 2016).

[5] Feger, T., Ireland, M. J., Schwab, C., Bento, J., Bacigalupo, C., and Coutts, D. W., "Attaining m s ${ }^{-1}$ level intrinsic Doppler precision with RHEA, a low-cost single-mode spectrograph," Experimental Astronomy 42, 285-300 (Dec 2016).

[6] Crepp, J. R., Crass, J., King, D., Bechter, A., Bechter, E., Ketterer, R., Reynolds, R., Hinz, P., Kopon, D., and Cavalieri, D., "iLocater: a diffraction-limited Doppler spectrometer for the Large Binocular Telescope," in [SPIE ], Society of Photo-Optical Instrumentation Engineers (SPIE) Conference Series 9908, 990819 (Aug 2016).

[7] Gibson, R. K., Oppenheimer, R., Matthews, C. T., and Vasisht, G., "Characterization of the C-RED 2: A High Frame Rate Near-Infrared Camera," arXiv e-prints, arXiv:1911.04567 (Nov 2019).

[8] Mawet, D., Fitzgerald, M., Konopacky, Q., Beichman, C., Jovanovic, N., Dekany, R., Hover, D., Chisholm, E., Ciardi, D., Artigau, É., Banyal, R., Beatty, T., Benneke, B., Blake, G. A., Burgasser, A., Canalizo, G., Chen, G., Do, T., Doppmann, G., Doyon, R., Dressing, C., Fang, M., Greene, T., Hillenbrand, L., Howard, A., Kane, S., Kataria, T., Kempton, E., Knutson, H., Kotani, T., Lafrenière, D., Liu, C., Nishiyama, S., Pand ey, G., Plavchan, P., Prato, L., Rajaguru, S. P., Robertson, P., Salyk, C., Sato, B., Schlawin, E., Sengupta, S., Sivarani, T., Skidmore, W., Tamura, M., Terada, H., Vasisht, G., Wang, J., and Zhang, H., "High-resolution Infrared Spectrograph for Exoplanet Characterization with the Keck and Thirty Meter Telescopes," in $[B A A S], \mathbf{5 1}, 134$ (Sep 2019).

[9] Plavchan, P., Cale, B., Newman, P., Hamze, B., Latouf, N., Matzko, W., Beichman, C., Ciardi, D., Purcell, B., and Lightsey, P., "EarthFinder: A Precise Radial Velocity Probe Mission Concept For the Detection of Earth-Mass Planets Orbiting Sun-like Stars," arXiv e-prints, arXiv:1803.03960 (Mar 2018).

[10] Cvetojevic, N., Lawrence, J. S., Ellis, S. C., Bland -Hawthorn, J., Haynes, R., and Horton, A., "Characterization and on-sky demonstration of an integrated photonic spectrograph for astronomy," Optics Express 17, 18643-18650 (Oct 2009).

[11] Jovanovic, N., Cvetojevic, N., Norris, B., Betters, C., Schwab, C., Lozi, J., Guyon, O., Gross, S., Martinache, F., and Tuthill, P., "Demonstration of an efficient, photonic-based astronomical spectrograph on an 8-m telescope," Optics Express 25, 17753 (Jul 2017d).

[12] Gatkine, P., Veilleux, S., and Dagenais, M., "Astrophotonic Spectrographs," Applied Sciences 9, 290 (Jan 2019).

[13] Cvetojevic, N., Jovanovic, N., Lawrence, J., Withford, M., and Bland-Hawthorn, J., "Developing arrayed waveguide grating spectrographs for multi-object astronomical spectroscopy," Optics Express 20, 2062 (Jan 2012a).

[14] Geuzebroek, D., van Rees, A., Klein, E., and Lawniczuk, K., "Ultra-wide band (400-1700nm) integrated spectrometer based on arrayed waveguide gratings for spectral tissue sensing," in [2017 IEEE 14th International Conference on Group IV Photonics (GFP)], 83-84 (Aug 2017).

[15] Bland-Hawthorn, J. and Horton, A., [Instruments without optics: an integrated photonic spectrograph], vol. 6269 of Society of Photo-Optical Instrumentation Engineers (SPIE) Conference Series, 62690N (2006).

[16] Bland-Hawthorn, J., Lawrence, J., Robertson, G., Campbell, S., Pope, B., Betters, C., Leon-Saval, S., Birks, T., Haynes, R., Cvetojevic, N., and Jovanovic, N., [PIMMS: photonic integrated multimode microspectrograph], vol. 7735 of Society of Photo-Optical Instrumentation Engineers (SPIE) Conference Series, $77350 \mathrm{~N}$ (2010).

[17] Cvetojevic, N., Jovanovic, N., Betters, C., Lawrence, J. S., Ellis, S. C., Robertson, G., and Bland -Hawthorn, J., "First starlight spectrum captured using an integrated photonic micro-spectrograph," $A \& A$ 544, L1 (Aug 2012b).

[18] Trinh, C. Q., Ellis, S. C., Bland -Hawthorn, J., Lawrence, J. S., Horton, A. J., Leon-Saval, S. G., Shortridge, K., Bryant, J., Case, S., and Colless, M., "GNOSIS: The First Instrument to Use Fiber Bragg Gratings for OH Suppression," AJ 145, 51 (Feb 2013).

[19] Leon-Saval, S. G., Argyros, A., and Bland-Hawthorn, J., "Photonic lanterns," Nanophotonics 2, 429-440 (Dec. 2013). 
[20] Birks, T. A., Gris-Sánchez, I., Yerolatsitis, S., Leon-Saval, S. G., and Thomson, R. R., "The photonic lantern," Adv. Opt. Photon. 7, 107-167 (Jun 2015).

[21] Jovanovic, N., Schwab, C., Guyon, O., Lozi, J., Cvetojevic, N., Martinache, F., Leon-Saval, S., Norris, B., Gross, S., and Doughty, D., "Efficient injection from large telescopes into single-mode fibres: Enabling the era of ultra-precision astronomy," A\&A 604, A122 (Aug 2017a).

[22] Lawrence, J., Bland-Hawthorn, J., Cvetojevic, N., Haynes, R., and Jovanovic, N., [Miniature astronomical spectrographs using arrayed-waveguide gratings: capabilities and limitations], vol. 7739 of Society of PhotoOptical Instrumentation Engineers (SPIE) Conference Series, 77394I (2010).

[23] Gatkine, P., Veilleux, S., Hu, Y., Bland -Hawthorn, J., and Dagenais, M., "Arrayed waveguide grating spectrometers for astronomical applications: new results," Optics Express 25, 17918 (Jul 2017).

[24] Zhu, T., Hu, Y., Gatkine, P., Veilleux, S., Bland-Hawthorn, J., and Dagenais, M., "Ultrabroadband high coupling efficiency fiber-to-waveguide coupler using $\mathrm{si}_{3} \mathrm{n}_{4} / \mathrm{sio}_{2}$ waveguides on silicon," IEEE Photonics Journal 8, 1-12 (Oct 2016).

[25] Zhu, T., Hu, Y., Gatkine, P., Veilleux, S., Bland-Hawthorn, J., and Dagenais, M., "Arbitrary on-chip optical filter using complex waveguide bragg gratings," Applied Physics Letters 108(10), 101104 (2016).

[26] Douglass, G., Dreisow, F., Gross, S., Nolte, S., and Withford, M. J., "Towards femtosecond laser written arrayed waveguide gratings," Opt. Express 23, 21392-21402 (Aug 2015).

[27] Douglass, G., Dreisow, F., Gross, S., and Withford, M. J., "Femtosecond laser written arrayed waveguide gratings with integrated photonic lanterns," Opt. Express 26, 1497-1505 (Jan 2018).

[28] Douglass, G., Arriola, A., Heras, I., Martin, G., Coarer, E. L., Gross, S., and Withford, M. J., "Novel concept for visible and near infrared spectro-interferometry: laser-written layered arrayed waveguide gratings," Opt. Express 26, 18470-18479 (Jul 2018).

[29] Cvetojevic, N., Development and Implementation of the Integrated Photonic Spectrograph for Astronomy, $\mathrm{PhD}$ thesis, Macquarie University (2013).

[30] Day, P. K., LeDuc, H. G., Mazin, B. A., Vayonakis, A., and Zmuidzinas, J., "A broadband superconducting detector suitable for use in large arrays," Nature 425, 817-821 (Oct 2003).

[31] Cropper, M., Barlow, M., Perryman, M. A. C., Horne, K., Bingham, R., Page, M., Guttridge, P., Smith, A., Peacock, A., Walker, D., and Charles, P., "A concept for a superconducting tunnelling junction based spectrograph," Monthly Notice of the Royal Astronomical Society 344, 33-44 (Sept. 2003).

[32] Szypryt, P., Meeker, S. R., Coiffard, G., Fruitwala, N., Bumble, B., Ulbricht, G., Walter, A. B., Daal, M., Bockstiegel, C., Collura, G., Zobrist, N., Lipartito, I., and Mazin, B. A., "Large-format platinum silicide microwave kinetic inductance detectors for optical to near-IR astronomy," Optics Express 25(21), 2589425909 (2017).

[33] Douglass, G., Dreisow, Gross, S., and Withford, M. J., "Femtosecond laser written arrayed waveguide gratings with integrated photonic lanterns," Opt. Express 26(2), 1497-1505 (2018). 